\title{
Personalized risk for clinical progression in cognitively normal subjects - the ABIDE project
}

Ingrid S. van Maurik ${ }^{1,2^{*}}$, Rosalinde E. R. Slot ${ }^{1}$, Sander C. J. Verfaillie', Marissa D. Zwan ${ }^{1}$, Femke H. Bouwman', Niels D. Prins ${ }^{1,3}$, Charlotte E. Teunissen ${ }^{4}$, Philip Scheltens ${ }^{1}$, Frederik Barkhof ${ }^{5,6}$, Mike P. Wattjes ${ }^{5}$, Jose Luis Molinuevo ${ }^{7}$, Lorena Rami ${ }^{7}$, Steffen Wolfsgruber ${ }^{8,9}$, Oliver Peters ${ }^{10}$, Frank Jessen ${ }^{11}$, Johannes Berkhof ${ }^{2}$, Wiesje M. van der Flier ${ }^{1,2}$ and for the Alzheimer's Disease Neuroimaging Initiative

\begin{abstract}
Background: Biomarkers such as cerebrospinal fluid (CSF) and magnetic resonance imaging (MRI) have predictive value for progression to dementia in patients with mild cognitive impairment (MCl). The pre-dementia stage takes far longer, and the interpretation of biomarker findings is particular relevant for individuals who present at a memory clinic, but are deemed cognitively normal. The objective of the current study is to construct biomarker-based prognostic models for personalized risk of clinical progression in cognitively normal individuals presenting at a memory clinic.

Methods: We included 481 individuals with subjective cognitive decline (SCD) from the Amsterdam Dementia Cohort. Prognostic models were developed by Cox regression with patient characteristics, MRI, and/or CSF biomarkers to predict clinical progression to $\mathrm{MCl}$ or dementia. We estimated 5- and 3-year individualized risks based on patient-specific values. External validation was performed on Alzheimer's Disease Neuroimaging Initiative (ADNI) and an European dataset.

Results: Based on demographics only (Harrell's C = 0.70), 5- and 3-year progression risks varied from 6\% [3-11] and 4\% [2-8] (age 55, MMSE 30) to 38\% [29-49] and 28\% [21-37] (age 70, MMSE 27). Normal CSF biomarkers strongly decreased progression probabilities (Harrell's $C=0.82$ ). By contrast, abnormal CSF markedly increased risk (5 years, 96\% [56-100]; 3 years, 89\% [44-99]). The CSF model could reclassify 58\% of the individuals with an "intermediate" risk (35-65\%) based on the demographic model. MRI measures were not retained in the models.
\end{abstract}

Conclusion: The current study takes the first steps in a personalized approach for cognitively normal individuals by providing biomarker-based prognostic models.

Keywords: Biomarkers, Progression, Cerebrospinal fluid, Magnetic resonance imaging

\section{Background}

Dementia disorders place a huge burden on society and are set to bulge due to an aging population [1,2]. Alzheimer's disease $(\mathrm{AD})$ is the most common cause of dementia and represents the largest unmet medical need in neurology [3]. In order to bring therapy and support to individuals as timely and accurate as possible, diagnostic tests play a key

\footnotetext{
*Correspondence: i.vanmaurik@vumc.nl

'Alzheimer Center Amsterdam, Department of Neurology, Amsterdam Neuroscience, Vrije Universiteit Amsterdam, Amsterdam UMC, Amsterdam, The Netherlands ${ }^{2}$ Department of Epidemiology and Biostatistics, Vrije Universiteit Amsterdam, Amsterdam UMC, Amsterdam, The Netherlands

Full list of author information is available at the end of the article
}

role. In individual patients with mild cognitive impairment (MCI), biomarkers such as cerebrospinal fluid (CSF) and magnetic resonance imaging (MRI) have been shown to have predictive value for progression to dementia [4]. However, the pre-dementia stage of $\mathrm{AD}$ takes far longer, as neuropathological changes already start in the cognitively normal stage $[3,5-8]$. For that reason, recent criteria proposed a biological framework for $\mathrm{AD}$ in which $\mathrm{AD}$ is classified based on the presence of pathology rather than the presence of clinical symptoms [8].

On a group level, AD biomarkers are predictive in cognitively normal individuals as well. For example, a reduced hippocampal volume on MRI has been associated 
with an increased risk of clinical progression [9-11]. Furthermore, an abnormal AD biomarker profile in CSF has been shown to be strongly associated with clinical progression [12-16]. Moreover, even relatively low amyloid $\beta 1-42$ (A $\beta$ ) levels, yet within the normal range, have been associated with clinical progression, indicating that simple dichotomous cutoffs fail to extract all information available in these markers and moreover may erroneously reassure individuals [17].

The question how these findings on group level translate to the individual is particular relevant for individuals who present with worries about their memory at a memory clinic, but are deemed cognitively normal. Unfortunately, findings on group level cannot be translated directly to the individual and the interpretation of biomarkers is not optimized. In addition, the meaning of biomarkers should ideally be interpreted in the context of an individual's own characteristics [18], but information on how to weigh and combine multiple sources of information is lacking. Therefore, clinicians are generally reluctant to disclose biomarker results to cognitively normal individuals. Nonetheless, individuals and caregivers become increasingly assertive, demanding more specific and individually tailored information [19].

The objective of this study was to optimize the interpretation of biomarkers by composing individualized prediction models for clinical progression to $\mathrm{MCI}$ or dementia based on MRI and/or CSF biomarkers that could be used in cognitively normal individuals.

\section{Methods}

\section{Participants}

We included 481 cognitively normal individuals from the Amsterdam Dementia Cohort (ADC) and ongoing SCIENCe project, with a baseline diagnosis of subjective cognitive decline (SCD), available baseline MMSE, and available baseline MRI and/or CSF data [20, 21]. All individuals had their baseline visit in our memory clinic between January 2000 and November 2015. Individuals with a diagnosis of MCI or dementia within 6 months after baseline were excluded from the analysis as they were likely to have been misclassified at baseline.

Baseline diagnostic work-up consisted of a standardized 1-day dementia screening [20,21]. Clinical diagnosis was made by consensus in a multi-disciplinary meeting. Individuals were labeled with SCD if they presented with cognitive complaints, had normal results on clinical assessments, and did not meet criteria for MCI, dementia, or any other neurologic or psychiatric disorder known to cause cognitive complaints (i.e., cognitively normal) [22].

Standardized annual follow-up included a follow-up visit with the neurologist and neuropsychologist, and diagnoses were re-evaluated in a multi-disciplinary meeting [20]. Until early 2012, MCI was diagnosed according to Petersen's criteria and from 2012 onwards, the diagnosis of MCI was based on National Institute on Aging-Alzheimer's Association (NIA-AA) criteria [23, 24]. The diagnosis of AD dementia and other types of dementia was based on international diagnostic or research consensus criteria [25-28].

\section{MRI}

Before 2008, brain MRI was performed on 1.0 and 1.5T MRI systems (Siemens Magnetom Avanto, Vision, Impact and Sonata, GE Healthcare Signa HDXT). From 2008 on, MRI of the brain was performed on 3T MRI systems (MR750, GE Medical Systems, Ingenuity TF PET/MR, Philips Medical Systems; Titan, Toshiba Medical Systems). The standard dementia protocol with whole brain coverage included near-isotropic sagittal 3D T1-weighted images (including oblique coronal reconstructions), sagittal 3D T2-weighted fluid-attenuated inversion recovery (FLAIR) (including axial reconstructions), axial T2-weighted turbo spin-echo, and axial T2*-weighted gradient echo sequence or alternatively SWI sequences. MRI data was available for $432(90 \%)$ individuals.

Bilateral hippocampal volume $(\mathrm{HCV}, \mathrm{mL})$ was estimated using FMRIBs Integrated Registration and Segmentation Tool (FIRST) [29]. Normalized brain volumes (NWBV, $\mathrm{mL}$ ) were estimated with SIENAX (Structural Image Evaluation using Normalization of Atrophy Cross-sectional) using optimized settings [30]. Additionally, visual rating of MRI was performed according to semi-quantitative visual rating scales for medial temporal lobe atrophy (MTA, 0-4) and global cortical atrophy (GCA, 0-3) [31, 32].

\section{CSF analysis}

CSF was obtained by lumbar puncture and collected in polypropylene tubes (Sarstedt, Nurmberg, Germany) and processed according to international guidelines [33]. CSF biomarkers Amyloid $\beta 1-42(\mathrm{~A} \beta)$ and total Tau (tau) were measured using sandwich enzyme-linked immuno sorbent assay (ELISA) on a routine basis (Innotest, Fujirebio, Gent, Belgium) [34]. Baseline CSF data was available for 344 $(72 \%)$ individuals.

\section{Statistical analysis}

All analyses were carried out in STATA 14SE. Prognostic models were constructed with Cox regression analysis (determinants as continuous measures; CSF biomarkers log-transformed). The models were constructed with complete cases only, and therefore, the number of individuals varied across models. No differences in demographic characteristic or baseline survival were found between individuals with complete data and incomplete data (Additional file 1: Table S1). The clinical end-point was MCI or dementia [23-28].

First, a prognostic model based on patient characteristics (age, gender, and MMSE) and interactions between the characteristics was constructed. Subsequently, we added 
either MRI biomarkers (volumetric measures: HCV, NWBV, or visual ratings: MTA, GCA), CSF biomarkers $(\mathrm{A} \beta, \mathrm{Tau})$, or both to the model. The models with volumetric MRI measures were adjusted for field strength. In all analyses, we intensively investigated main effects of patient characteristics and interaction effects between biomarkers and between biomarker and patient characteristics. Effects were retained in the model via a backward selection procedure, if $p$ value $\leq .10$. The prognostic accuracy of the model was measured by Harrell's $C$-statistic.

We estimated cumulative progression probabilities with 95\% confidence intervals using the survci command in STATA [35]. We report 5-, 3-, and 1-year cumulative progression probabilities with corresponding confidence intervals. Since the clinical follow-up visit times showed some variation, the cutoff for 1-year follow-up was set at 1.5 years, for 3 -year follow-up at 3.5 years, and for 5 -year follow-up at 5.5 years. As an example, we provide risk estimates for individuals with an age of 55 and 70; females and males and MMSE scores of 30 or 27. To contrast individuals with normal and abnormal MRI and CSF results, we entered 10th and 90th percentile MRI and CSF values in the Cox model. Note that when using the models, any value can be entered for a variable. Based on the constructed models, 5-year progression probabilities were calculated for every patient in the cohort. Subsequently, we labeled each individual as having low risk $(\leq 35 \%)$, intermediate risk (35-65\%), or high risk (>65\%).

In an additional set of analyses, we repeated all analyses to construct models predicting progression to $\mathrm{MCI}$ or AD dementia as clinical end-point. In this set of analyses, individuals progressing to non-AD were censored at time of diagnosis of non-AD dementia.

\section{Validation}

We internally validated the models by five-fold cross-validation, in which we again applied a backward selection procedure.

Next, we performed external validation of our models on a sample comprising individuals with SCD from Alzheimer's Disease Neuroimaging Initiative (ADNI, $n=92$ ), Dementia Competence Network (DCN, $n=86$ ), and Barcelona Memory Clinic $(n=41)$. Like the ADC cohort, DCN and Barcelona included individuals that went to the memory clinic to seek medical help and were labeled with SCD when cognitive testing could not confirm their cognitive complaints, and criteria for $\mathrm{MCI}$, dementia, or other neurological or psychological diseases were not met. ADNI on the other hand is a population-based study. Subjects were labeled with SCD when a significant subjective memory concern was reported by the subject, informant, or clinician.

CSF was measured with Innotest in the DCN and Barcelona cohort and with Elecsys in ADNI. Therefore, biomarker values were standardized for the analysis to remove measurement levels. Patient characteristics from the cohorts can be found in Additional file 1: Table S2. Differences between the cohorts included a higher age in the ADNI cohort, higher progression rates in the DCN, longer follow-up for ADC and Barcelona individuals; and ADNI and Barcelona individuals were more often female (Additional file 1: Table S2). Established models were fitted to the validation data, and Harrell's $C$-statistics were calculated.

\section{Results}

During a mean follow-up of $3 \pm 2$ years, 70 (15\%) individuals showed clinical progression to MCI $(n=49), \mathrm{AD}$ dementia $(n=10)$, or non-AD dementia $(n=11)$. Mean age was $62 \pm 9$ years, $211(44 \%)$ of the individuals were female, and the mean MMSE score was $28 \pm 1.6$ (Table 1).

Table 2 shows the variables and corresponding coefficients included in the models (demographics only, CSF model, MRI volumetric model, and MRI visual model).

The demographics only model included age and MMSE (sex not included, $p$ value $>.10$ ). Harrell's $C$-statistic was 0.70 (Table 2). Younger individuals (as an example, we set age at 55) with MMSE-scores of 30 had a low risk of progression: after 5 years $6 \%$ [3-11], after 3 years $4 \%[2-8]$, and after 1 year $2 \%[0-2]$. On the other end of the spectrum, older individuals (70) with lower MMSE-scores (27) had higher progression probabilities; risk of progression after 5 years was $38 \%$ [29-49], after 3 years 28\% [21-37], and after 1 year $11 \%$ [7-16] (Table 3).

Table 1 Baseline characteristics

\begin{tabular}{ll}
\hline & SCD individualsn =481 \\
\hline No. (\%) with clinical progression & $70(15 \%)$ \\
Progression to MCl & $49(10 \%)$ \\
Progression to non-AD dementia & $10(2 \%)$ \\
Age & $11(2 \%)$ \\
Gender, no. (\%) females & $62 \pm 9$ \\
MMSE & $211(44 \%)$ \\
Follow-up duration & $28 \pm 1.6$ \\
Medial temporal lobe atrophy & $3 \pm 2$ \\
Global cortical atrophy & $0.4 \pm 0.5$ \\
Hippocampal volume (cm3) & $0.4 \pm 0.6$ \\
Normalized whole brain volume (cm $\left.{ }^{3}\right)$ & $7.2 \pm 1$ \\
Amyloid $\beta 1-42$ & $1453 \pm 100$ \\
Total Tau & $879 \pm 260$ \\
p-tau & $298 \pm 196$ \\
\hline Pata are mean & $49 \pm 22$ \\
\hline
\end{tabular}

Data are mean \pm standard deviation, unless otherwise specified. MMSE minimental state examination, MRI magnetic resonance imaging, CSF cerebrospinal fluid 
Table 2 Regression coefficient of the final model

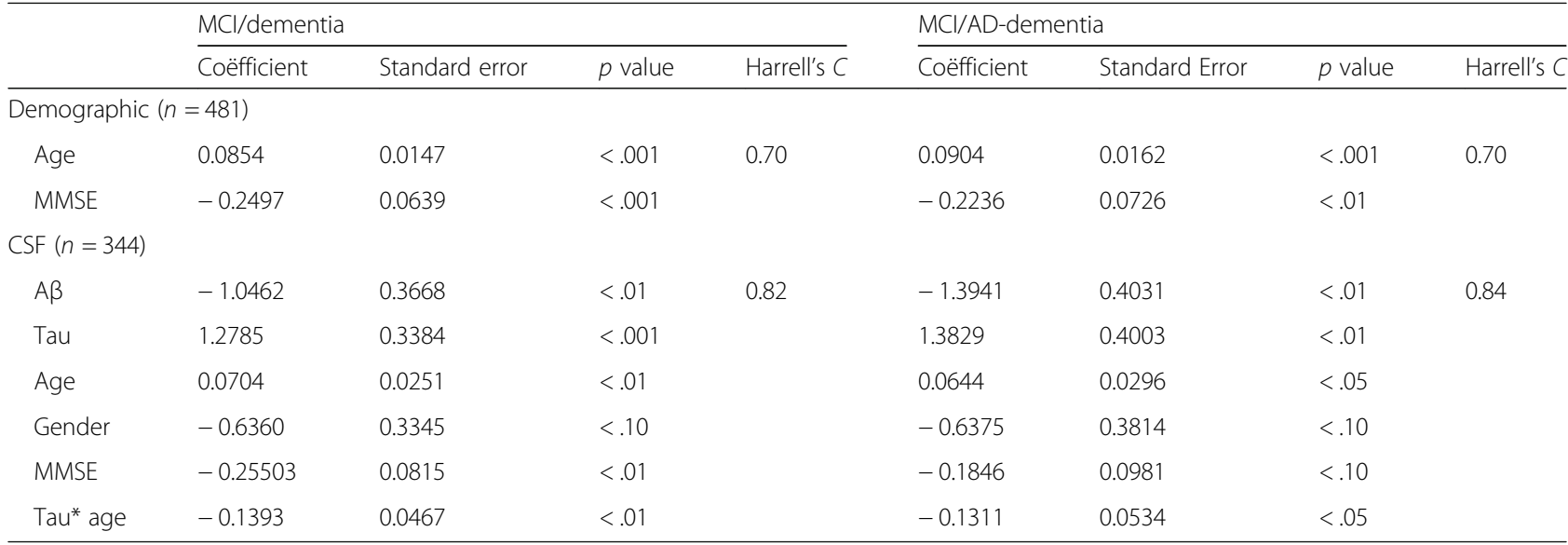

CSF biomarkers ( $\mathrm{A} \beta$ and Tau) are log-transformed and centered. MMSE mini-mental state examination, Tau* age interaction between age and Tau. Interaction term was centered and standardized to allow inclusion in the model

Table 3 Progression probabilities after 1 year, 3 years, and 5 years

\begin{tabular}{|c|c|c|c|c|c|c|c|c|}
\hline & \multirow[b]{2}{*}{ Age } & \multirow[b]{2}{*}{ Sex } & \multirow[b]{2}{*}{ MMSE } & \multirow[t]{2}{*}{ Demographics only } & \multicolumn{4}{|l|}{ CSF } \\
\hline & & & & & Normal & AB abnormal & Tau abnormal & Both abnormal \\
\hline \multirow[t]{8}{*}{1 year } & \multirow[t]{4}{*}{55} & \multirow[t]{2}{*}{$\mathrm{m}$} & 30 & $2 \%[0-2]$ & $0 \%[0-1]$ & $0 \%[0-2]$ & $12 \%[3-38]$ & $26 \%[7-71]$ \\
\hline & & & 27 & $3 \%[2-5]$ & $0 \%[0-2]$ & $0 \%[1-5]$ & $26 \%[8-65]$ & $51 \%$ [18-92] \\
\hline & & \multirow[t]{2}{*}{$f$} & 30 & $2 \%[0-2]$ & $0 \%[0-1]$ & $0 \%[0-1]$ & $6 \%[2-23]$ & $15 \%[4-48]$ \\
\hline & & & 27 & $3 \%[2-5]$ & $0 \%[0-1]$ & $0 \%[1-3]$ & $15 \%[4-44]$ & $32 \%[9-77]$ \\
\hline & \multirow[t]{4}{*}{70} & \multirow[t]{2}{*}{$\mathrm{m}$} & 30 & $5 \%[3-9]$ & $5 \%[2-15]$ & $13 \%[5-34]$ & $4 \%[1-11]$ & $8 \%[3-20]$ \\
\hline & & & 27 & $11 \%[7-16]$ & $12 \%[5-30]$ & $29 \%$ [11-64] & $9 \%[3-21]$ & $19 \%[9-36]$ \\
\hline & & \multirow[t]{2}{*}{$f$} & 30 & $5 \%[3-9]$ & $3 \%$ [1-9] & $7 \%[2-22]$ & $3 \%[1-6]$ & $5 \%[2-12]$ \\
\hline & & & 27 & $11 \%[7-16]$ & $7 \%[2-22]$ & $16 \%[5-46]$ & $5 \%[2-12]$ & $10 \%[4-24]$ \\
\hline \multirow[t]{8}{*}{3 years } & \multirow[t]{4}{*}{55} & \multirow[t]{2}{*}{$\mathrm{m}$} & 30 & $4 \%[2-8]$ & $0 \%[0-2]$ & $1 \%[1-6]$ & $32 \%[10-78]$ & $60 \%[20-98]$ \\
\hline & & & 27 & $9 \%[6-14]$ & $1 \%[0-5]$ & $2 \%[0-12]$ & $60 \%[23-95]$ & $89 \%$ [44-99] \\
\hline & & \multirow[t]{2}{*}{$f$} & 30 & $4 \%[2-8]$ & $0 \%[0-1]$ & $1 \%[0-3]$ & $19 \%[5-55]$ & $39 \%[11-87]$ \\
\hline & & & 27 & $9 \%[6-14]$ & $0 \%[0-3]$ & $1 \%[0-7]$ & $39 \%[12-84]$ & $69 \%$ [26-99] \\
\hline & \multirow[t]{4}{*}{70} & \multirow[t]{2}{*}{$\mathrm{m}$} & 30 & $15 \%[10-21]$ & $16 \%[6-39]$ & $35 \%$ [14-72] & $13 \%$ [4-27] & $23 \%$ [11-46] \\
\hline & & & 27 & $28 \%[21-37]$ & $33 \%$ [14-71] & $64 \%$ [30-96] & $24 \%$ [10-49] & $48 \%[28-71]$ \\
\hline & & \multirow[t]{2}{*}{$f$} & 30 & $15 \%[10-21]$ & $9 \%[3-25]$ & $21 \%[7-53]$ & $7 \%[3-16]$ & $16 \%[6-29]$ \\
\hline & & & 27 & $28 \%[21-37]$ & $21 \%$ [7-53] & $43 \%$ [15-85] & $14 \%[5-32]$ & $29 \%$ [14-50] \\
\hline \multirow[t]{8}{*}{5 years } & \multirow[t]{4}{*}{55} & \multirow[t]{2}{*}{$\mathrm{m}$} & 30 & $6 \%[3-11]$ & $1 \%[0-3]$ & $1 \%[0-8]$ & $43 \%$ [13-89] & $73 \%$ [27-98] \\
\hline & & & 27 & $12 \%[8-20]$ & $1 \%[1-7]$ & $4 \%[1-17]$ & $73 \%$ [30-99] & $96 \%$ [56-100] \\
\hline & & \multirow[t]{2}{*}{$f$} & 30 & $6 \%[3-11]$ & $0 \%[0-2]$ & $1 \%[0-4]$ & $25 \%$ [7-69] & $51 \%$ [16-94] \\
\hline & & & 27 & $12 \%[8-20]$ & $1 \%[0-4]$ & $2 \%[0-10]$ & $50 \%$ [17-93] & $81 \%$ [35-98] \\
\hline & \multirow[t]{4}{*}{70} & \multirow[t]{2}{*}{$\mathrm{m}$} & 30 & $20 \%$ [14-29] & $22 \%$ [9-51] & $47 \%$ [19-84] & $17 \%[6-36]$ & $32 \%[16-58]$ \\
\hline & & & 27 & $38 \%$ [29-49] & $44 \%$ [19-83] & $78 \%$ [39-98] & $32 \%[24-62]$ & $60 \%$ [38-83] \\
\hline & & \multirow[t]{2}{*}{$f$} & 30 & $20 \%$ [14-29] & $13 \%[4-34]$ & $28 \%$ [10-66] & $10 \%[5-22]$ & $21 \%[8-38]$ \\
\hline & & & 27 & $38 \%$ [29-49] & $28 \%[10-66]$ & $55 \%$ [20-94] & $20 \%$ [10-39] & $39 \%[20-66]$ \\
\hline
\end{tabular}


When we evaluated MRI markers, neither volumetric nor visual measures added predictive value over the demographic model including age and MMSE ( $p$ value $>$.10). In the CSF model, female gender, higher age, lower MMSE score, lower $A \beta$, and higher Tau values were predictive of progression. Moreover, an interaction between Tau and age is retained in the model. Tau was more predictive than $A \beta$, especially in younger individuals (Tau* age $p$ value <.01, Table 3 and Fig. 1). Harrell's $C$-statistic was 0.82 (Table 2, similar when $\mathrm{p}$-tau was included instead of tau (Additional file 1: Table S3). To contrast individuals with normal and abnormal CSF results, we derived probabilities for individuals with 10th and 90th percentile CSF values from the model.

Abnormal $A \beta$ and Tau resulted in high 5-, 3-, and 1-year progression risks; 96\% [56-100], 89\% [44-99], and 51\% [18-92]. By contrast, normal CSF biomarkers strongly decreased progression probabilities to $1 \%[0-3]$ in 5 year, $0 \%[0-2]$ in 3 years, and $0 \%[0-1]$ in 1 year, indicating the negative predictive value of these biomarkers. Please note that we report examples, as the model provides risks for any given value.

Figure 2 shows the distribution of 5 -year progression probabilities based on the model including CSF biomarkers. The majority of individuals, $84 \%(n=$ 290) were labeled as having low risk of progression, $12 \%(n=41)$ had intermediate risk of progression, and $4 \%(n=13)$ had high risk of progression. Of note, $58 \%$ of the individuals that were classified as "intermediate" based on the demographic model could be reclassified as having low (49\%) or high (9\%) risk according to the CSF model (Additional file 1: Table S4).

In an additional set of analyses, we repeated the analysis restricting the outcome to progression to $\mathrm{MCI}$ or AD dementia. The prognostic accuracy of the CSF model increased in line with specificity for $\mathrm{AD}$ of the biomarkers under evaluation. Harrell's $C$-statistic remained 0.70 for the demographic model and increased to 0.84 for the CSF model.

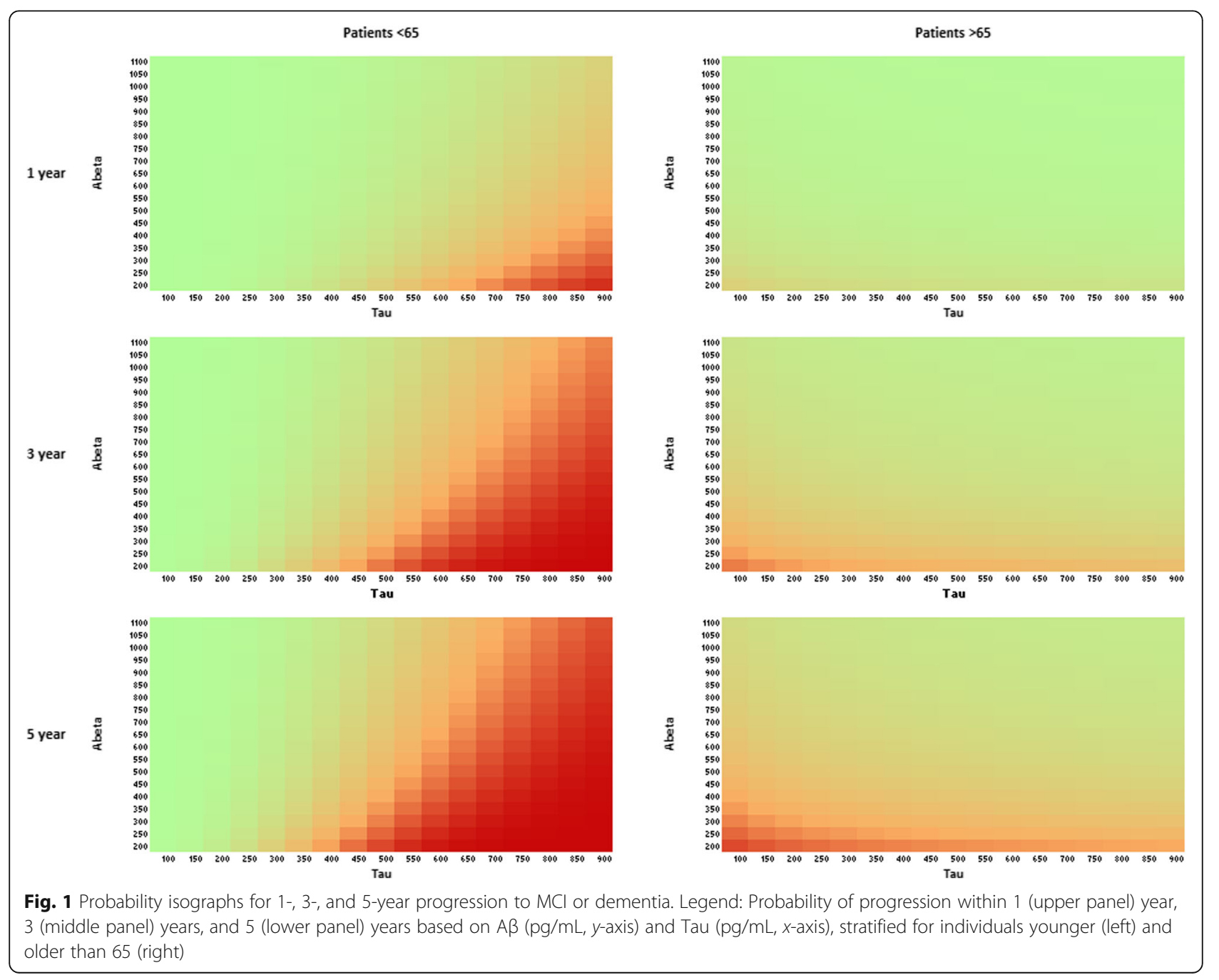




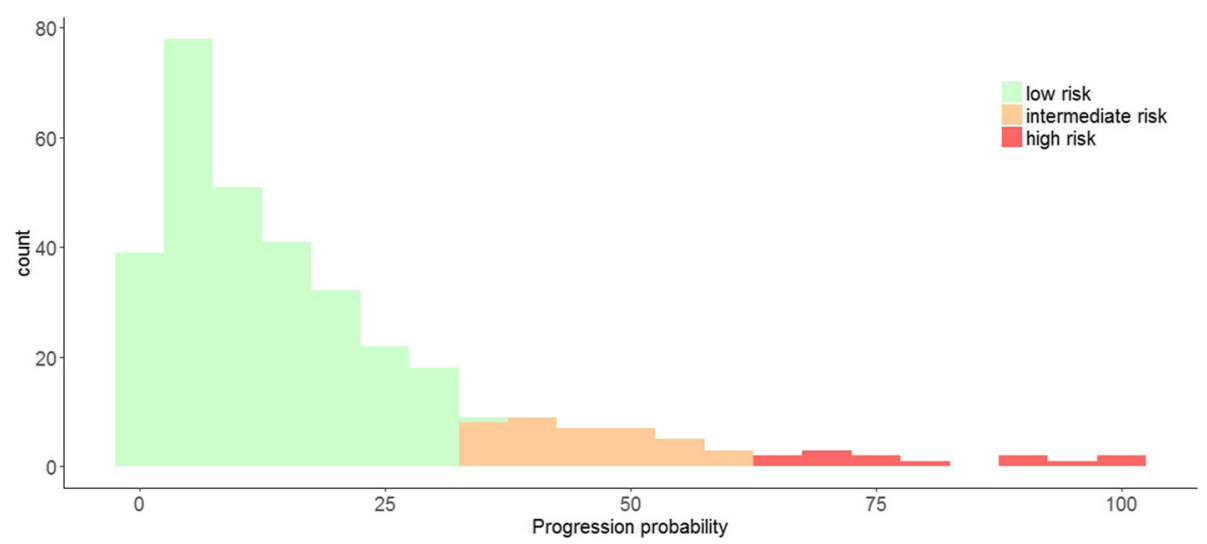

Fig. 2 Distribution of 5-year progression probabilities. Legend: Distribution of 5-year progression probabilities based on the CSF model. Green: low risk 0-35\%; orange: intermediate 35-65\%; red: high risk 65-100\%

Internal validation by five-fold cross-validation confirmed prognostic performance in both models (Additional file 1: Table S5); cross-validation of the demographic model resulted in Harrell's $C$-statistics ranging from 0.63-0.77. The model with CSF biomarkers showed cross-validated Harrell's $C$ ranging from $0.75-0.90$.

External validation showed moderate performance of the models (demographic model, Harrell's $C=0.62$; CSF model, $C=0.68)$.

\section{Discussion}

In this study, we constructed biomarker prediction models that provide individual risk estimates of clinical progression in order to optimize the interpretation of biomarkers for cognitively normal SCD individuals. CSF biomarkers considerably improved prognostic performance over the use of age and MMSE only. This was mostly driven by their strong negative predictive value.

Alzheimer pathology as reflected in biomarker changes presumably starts more than 20 years before the onset of dementia [3, 8]. Clinicians are reluctant to disclose biomarker results to cognitively normal individuals presenting at a memory clinic, as former findings that were based on group level cannot directly be translated to the individual. Moreover, there is always a degree of uncertainty associated with the interpretation of biomarkers. With our models, we provide a first step towards a framework for a personalized approach, allowing the use of biomarker results for cognitively normal individuals presenting at memory clinics. This can be useful, as individuals and caregivers become increasingly assertive in their need for information on their risk of dementia. Moreover, interest in individualized risk profiling and both primary and secondary prevention strategies is increasing rapidly. Although truly longitudinal data are lacking, our sample allowed to infer predictions of progression over periods of 3 and even 5 years, which has great relevance for individuals and their family members. Probabilities of progression within 1 year in SCD individuals remain low, and this is in line with the notion that outcome at 1-year follow-up is not a reasonable time frame for SCD, as these individuals initially perform cognitively normal.

Former studies have shown the clinical relevance of CSF biomarkers in pre-dementia individuals on a group level $[15,16]$. In the current study, we found that Tau was a stronger predictor than A $\beta$. Particularly, Tau was more predictive for progression in younger individuals. Abnormal Tau values in older individuals were less predictive, probably due to normal aging processes or multiple pathologies in older individuals [36]. Moreover, gender was included as a predictor in the CSF model, meaning that CSF measures should also be interpreted in the context of a patient's gender. This fits with findings from a recently published review that showed the importance of sex differences for patient stratification and personalized treatment [37]. With these CSF biomarkers and patient characteristics, $88 \%$ of the individuals could be classified as having a high $(>65 \%)$ or low $(\leq 35 \%)$ risk of clinical progression within 5 years.

Former studies on MRI biomarkers have reported that cognitively normal individuals with SCD had lower hippocampal volumes compared to healthy controls [11]. Moreover, hippocampal atrophy and lower brain volumes predicted of progression to MCI and/or dementia [10, 38]. However, these previously reported significant results for MRI were mostly based on small absolute differences between groups of individuals, precluding their usefulness in individualized risk predictions. In the current study, MRI markers did not improve personalized risk estimates over the use of age and MMSE only. The effects of MRI biomarkers lost significance, as soon as age was included in the model, suggesting that the observed atrophy in this population is largely attributable to aging and/or did not 
capture additional predictive value over subtle cognitive impairment.

In a former study in MCI, we found that MRI biomarkers in combination with patient characteristics and also CSF biomarkers improve individualized prediction of progression to dementia [4]. The finding that atrophy on MRI has less predictive value in cognitively normal individuals than in MCI patients is consistent with the hypothetical biomarker model which suggests that CSF biomarker changes precede MRI-based estimates of neurodegeneration [39].

Among the limitations, we found that the models showed somewhat less prognostic performance in external cohorts. This may be attributable to the fact that the outcome in the current study is clinical progression to $\mathrm{MCI}$ or dementia. While dementia is a relatively definitive end-point, $\mathrm{MCI}$ patients may still remain stable or convert to normal states of cognition and variability in this diagnosis between centers may be larger than in case of dementia [3, 24, 40]. In addition, external validation is highly dependent on the case-mix of a sample. In the field of $\mathrm{SCD}$, one of the most important unresolved challenges is the variability of defining SCD across studies [41-43]. In the ADC, DCN, and Barcelona cohort, all individuals went to the memory clinic seeking help and their complaints might be more severe than in the population-based ADNI cohort. In ADNI, individuals were labeled with SCD when a significant subjective memory concern was reported by the subject, informant, or clinician. Moreover, the standardized diagnostic work-up differed between the centers. For example, ADNI measured CSF with Elecsys instead of Innotest and brain volume with Freesurfer software instead of FSL FIRST. However, we limited these differences as much as possible by standardizing the biomarker values to remove measurement levels.

Another limitation is that we used different scanners and field strengths. This however resembles real-life clinical practice, and we included field strength as an additional determinant in the models. Field strength, however, did not improve predictive ability of MRI models. In addition, we used MMSE as a measure of global cognition in our models, which has been described as an insensitive instrument in preclinical stages. Other measures for cognition (for example, a composite score of specific items of the MMSE and Clinical Dementia Rating scale; ADCOMS [44], or instrumental activities of daily living (IADL [45])) with higher sensitivity could improve the models. Such an approach might be subject for future studies. Lastly, the follow-up duration varied between individuals and the mean follow-up period of $3 \pm 2$ years was rather short in comparison with the assumed duration of the stage of preclinical AD. Nonetheless, we had enough power to estimate risks over a period of 5 years, which is a considerable duration of follow up.
A major strength of this study is the simplicity of the models. Often, the goal of constructing prediction models is to derive the most optimal combination of many variables. However, such models often require multiple pieces of data that are not easily available, consequently limiting their clinical footprint [46]. In the current paper, we took a different approach as we aimed to optimize the interpretation of MRI measures and CSF biomarkers in individuals with SCD: given that a clinician has decided to obtain MRI and/or CSF biomarkers in an individual with SCD with a given age, sex, and MMSE, this clinician wants to make optimal use of the results of MRI and CSF. By doing so, our models helps to interpret biomarkers in the individual patient (hence personalized) and shows proof of principle that personalized predictions could be feasible in very early stages of $\mathrm{AD}$. Another strength is that we used a large sample of SCD individuals. All individuals had an extensive screening at baseline to rule out MCI or other neurological causes of memory complaints and careful follow-up, with diagnosis re-evaluated in a multidisciplinary setting, which has contributed to the robustness of the data. The vast majority of individuals came to the memory clinic with worries about their cognitive functioning, rendering our models highly relevant for this population. In fact, this population is comparable to what in the new Alzheimer research framework is described as clinical stage 2 [8]. Our results confirm clinical validity of such stage 2 , as the presence of Alzheimer biomarkers strongly increases the risk of future progression to $\mathrm{MCI}$ or dementia. Another strong aspect of this study is that we accompany predictions with confidence intervals, which gives a good indication on precision of the prediction.

In an earlier study on communication of diagnostic test results, individuals and caregivers who recently visited a memory clinic indicated that they wanted more information on their prognosis and what test results meant for their personal lives ("what do these results mean for my future") [47]. Nonetheless, clinicians tend to be reluctant to disclose biomarker results to cognitively normal individuals. The major concern is that disclosure could increase anxiety [48]. An argument against the disclosure of risk is the lack of treatment options. But this raises the question whether it is ethical to withhold individuals from information that is actually available. Moreover, our models show that particularly the negative predictive value of the models is good, suggesting that biomarker results can be especially valuable to reassure patients.

\section{Conclusions}

In conclusion, we constructed prognostic models that allow interpretation of biomarker data in cognitively normal individuals in a memory clinic at the individual 
level. In light of future disease-modifying drugs, risk prediction on an individual level becomes increasingly important [49]. By integrating biomarker results and demographic characteristics in $\mathrm{AD}$ risk modeling, the current study takes the first steps in a personalized approach for cognitively normal individuals $[48,50]$. This is especially valuable for the reassurance of individuals with normal biomarkers, since clinical progression over a period of 5 years is very unlikely for them.

\section{Additional file}

Additional file 1: Figure S1. Flowchart patient selection. Figure S2.

Kaplan-Meier based on CSF risk group. Table S1. Demographic characteristics and baseline survival for complete cases and incomplete cases. Table S2. Baseline patient characteristics validation cohort. Table S3. Regression coefficient of CSF model including p-tau. Table S4. Reclassification table based on the demographic and CSF model. Table S5. Model development by internal cross validation. Table S6. Model development in the validation cohort. (DOCX $84 \mathrm{~kb}$ )

\section{Acknowledgements}

Research of the Alzheimer center Amsterdam is part of the neurodegeneration research program of Amsterdam Neuroscience. The Alzheimer Center Amsterdam is supported by Stichting Alzheimer Nederland and Stichting VUmc fonds. The clinical database structure was developed with funding from Stichting Dioraphte. Wiesje van der Flier holds the Pasman chair and is recipient of a grant by Stichting LSH-TKI (ABIDE-communication: LSHM16025). Ingrid van Maurik is appointed at a ZonMWMemorabel grant (ABIDE; project No 733050201), in the context of the Dutch Deltaplan Dementie. The Euro-SCD project has been funded by the EU Joint Programme - Neurodegenerative Disease Research (JPND_PS_FP-689-019). DCN has been funded by a grant from the German Federal Ministry of Education and Research (BMBF): Kompetenznetz Demenzen (01Gl0420). FB is supported by the NIHR UCLH Biomedical Research Centre. Sander Verfaillie and Rosalinde Slot are supported by a research grant from Gieske-Strijbis Fonds. Data used in preparation of this article were obtained from the Alzheimer's Disease Neuroimaging Initiative (ADNI) database (adni.loni.usc.edu). As such, the investigators within the ADNI contributed to the design and implementation of ADNI and/or provided data but did not participate in analysis or writing of this report. A complete listing of ADNI investigators can be found at: https://adni.loni.usc.edu/wp-content/uploads/how_to_apply/ ADNI_Acknowledgement_List.pdf.

\section{Funding}

This study was funded by Stichting LSH-TKI (ABIDE-communication: LSHM16025), which is co-financed by Alzheimer Nederland, Piramal Neuroimaging, VU University Medical Center and Amsterdam Medical Center.

\section{Availability of data and materials}

The corresponding author can provide the dataset used and/or the analysis performed upon reasonable request.

\section{Authors' contributions}

ISM, WMF and JB designed the study. RERS, SCJV, FHB, NDP, CET, PS, FB, MPW, JLM, LR, SW, OP and FJ collected the data. ISM and JB analyzed the data. ISM, WMF and JB interpreted the data and wrote the manuscript. SV, MDZ, FHB, NDP, CET, PS, FB, MPW, JLM, SW, OP, and FJ revised the manuscript. All authors read and approved the final manuscript.

\section{Ethics approval and consent to participate}

The study was approved by the local Medical Ethical Committee. All patients provided written informed consent for their clinical data to be used for research purposes.

\section{Consent for publication}

Not applicable.

\section{Competing interests}

Dr. Prins serves on the advisory board of Boehringer Ingelheim and Probiodrug and on the DSMB of Abbvie's M15-566 trial. He has provided consultancy services for Sanofi, Takeda, and Kyowa Kirin Pharmaceutical Development. NDP is the CEO and co-owner of the Alzheimer Research Center, Amsterdam, the Netherlands.

C. Teunissen serves on the advisory board of Fujirebio and Roche, received research consumables from Euroimmun, IBL, Fujirebio, Invitrogen, and Mesoscale Discovery; performed contract research for IBL, Shire, Boehringer, Roche, and Probiodrug; and received grants from the European Commission, the Dutch Research Council (ZonMW), Association of Frontotemporal Dementia/Alzheimer's Drug Discovery Foundation, ISAO, and the Alzheimer's Drug Discovery Foundation.

Dr. Scheltens has acquired grant support (for the institution) from GE Healthcare, Danone Research, Piramal, and MERCK. In the past 2 years, he has received consultancy/speaker fees (paid to the institution) from Lilly, GE Healthcare, Novartis, Sanofi, Nutricia, Probiodrug, Biogen, Roche, Avraham, and EIP Pharma.

Dr. Barkhof serves as a consultant for Biogen-Idec, Janssen Alzheimer Immunotherapy, Bayer-Schering, Merck-Serono, Roche, Novartis, Genzume, and Sanofi-aventis; has received sponsoring from EU-H2020, NIHR-UCLH, NOW, SMSR, TEVA, Novartis, Toshiba; and serves on the editorial boards of Radiology, Brain, Neuroradiology, MSJ, and Neurology.

Dr. Peters is on scientific advisory boards for Roche, Kyowa Kirin, Novartis, Lilly, and Piramal. He has received funding for travel or speaker honoraria from GSK, Nutricia, and Merck Serono. He has acted as a consultant for Affiris and Roche. He has received research support from Affiris, Piramal, BMS, Eli Lilly, Pfizer, Servier, TRX Pharmaceuticals, Lundbeck, and Genentech. Dr. Jessen has received consultation board honoraria and speakers fees from AC Immune, Lilly, GE Healthcare, Janssen, USB, Schwabe, Esai, Pfizer, Novartis, and Roche. He has received a research grant paid to his institution from Schwabe.

Dr. van der Flier performs contract research for Biogen MA Inc. Research programs of W.M.v.d.F. have been funded by ZonMW, NWO, EU-FP7, EUJPND, Alzheimer Nederland, Cardiovasculair Onderzoek Nederland, Stichting Dioraphte, Gieskes-Strijbis Fonds, Boehringer Ingelheim, Piramal Neuroimaging, Roche BV, Janssen Stellar, and Combinostics. All funding is paid to her institution.

van Maurik, Slot, Verfaillie, Dr. Zwan, Dr. Bouwman, Dr. Wattjes, Dr. Wolfsgruber, and Dr. Berkhof declare that they have no competing interests.

\section{Publisher's Note}

Springer Nature remains neutral with regard to jurisdictional claims in published maps and institutional affiliations.

\section{Author details \\ ${ }^{1}$ Alzheimer Center Amsterdam, Department of Neurology, Amsterdam Neuroscience, Vrije Universiteit Amsterdam, Amsterdam UMC, Amsterdam, The Netherlands. ${ }^{2}$ Department of Epidemiology and Biostatistics, Vrije Universiteit Amsterdam, Amsterdam UMC, Amsterdam, The Netherlands. 'Brain Research Center, Amsterdam, The Netherlands. ${ }^{4}$ Neurochemistry Laboratory and Biobank, Department of Clinical Chemistry, Amsterdam Neuroscience, Vrije Universiteit Amsterdam, Amsterdam UMC, Amsterdam, The Netherlands. ${ }^{5}$ Department of Radiology and Nuclear Medicine, Amsterdam Neuroscience, Vrije Universiteit Amsterdam, Amsterdam UMC, Amsterdam, The Netherlands. Institutes of Neurology and Healthcare Engineering, UCL, London, UK ${ }^{7}$ Alzheimer's Disease and Other Cognitive Disorders Unit, Neurology Service, Hospital Clínic, Barcelona, Spain and Institut d'Investigacions Biomèdiques August Pi i Sunyer (IDIBAPS), Barcelona, Spain. ${ }^{8}$ Department of Psychiatry and Psychotherapy, University of Bonn, Bonn, Germany. ${ }^{9}$ German Center for Neurodegenerative Diseases, Bonn, Germany. ${ }^{10}$ Department of Psychiatry, Charité Berlin, Campus Benjamin Franklin, Berlin, Germany. "Department of Psychiatry, University of Cologne, Cologne, Germany.}

Received: 14 September 2018 Accepted: 29 March 2019 Published online: 16 April 2019

\section{References}

1. Prince $M$, Anders M, Guerchet $M$, et al. The global impact of dementia. An analysis of prevalence, incidence, cost and trends. London: Published by Alzheimer's Disease International (ADI); 2015. 
2. Organization WH. Dementia: a public health priority. Geneva: World Health Organization; 2012.

3. Scheltens P, Blennow K, Breteler MM, et al. Alzheimer's disease. Lancet. 2016; 388(10043):505-17.

4. van Maurik IS, Zwan MD, Tijms B, et al. Interpreting biomarker results in individual $\mathrm{MCl}$ patients - the ABIDE project. JAMA Neurology. 2017;74(12): 1481-91.

5. Reiman EM, Quiroz YT, Fleisher AS, et al. Brain imaging and fluid biomarker analysis in young adults at genetic risk for autosomal dominant Alzheimer's disease in the presenilin 1 E280A kindred: a case-control study. Lancet Neurol. 2012;11(12):1048-56.

6. Morris JC. Early-stage and preclinical Alzheimer disease. Alzheimer Dis Assoc Disord. 2005:19(3):163-5.

7. Sperling RA, Aisen PS, Beckett LA, et al. Toward defining the preclinical stages of Alzheimer's disease: recommendations from the National Institute on Aging-Alzheimer's Association workgroups on diagnostic guidelines for Alzheimer's disease. Alzheimers Dement. 2011;7(3):280-92.

8. Jack C, Bennett DA, Blennow K, et al. NIA-AA research framework: toward a biological definition of Alzheimer's disease. Alzheimers Dement. 2018;14 535-62.

9. Scheef L, Spottke A, Daerr M, et al. Glucose metabolism, gray matter structure, and memory decline in subjective memory impairment. Neurology. 2012;79(13):1332-9.

10. Verfaillie SC, Tijms B, Versteeg A, et al. Thinner temporal and parietal cortex is related to incident clinical progression to dementia in patients with subjective cognitive decline. Alzheimers Dement (Amst). 2016;5:43-52

11. Jessen $F$, Feyen $L$, Freymann $K$, et al. Volume reduction of the entorhinal cortex in subjective memory impairment. Neurobiol Aging. 2006;27(12):1751-6.

12. Sierra-Rio A, Balasa M, Olives J, et al. Cerebrospinal fluid biomarkers predict clinical evolution in patients with subjective cognitive decline and mild cognitive impairment. Neurodegener Dis. 2016;16(1-2):69-76.

13. Hessen E, Nordlund A, Stalhammar J, et al. T-tau is associated with objective memory decline over two years in persons seeking help for subjective cognitive decline: a report from the Gothenburg-Oslo $\mathrm{MCl}$ study. J Alzheimers Dis. 2015;47(3):619-28.

14. Sun $Y$, Yang FC, Lin CP, Han Y. Biochemical and neuroimaging studies in subjective cognitive decline: progress and perspectives. CNS Neurosci Ther. 2015;21(10):768-75.

15. Wolfsgruber S, Polcher A, Koppara A, et al. Cerebrospinal fluid biomarkers and clinical progression in patients with subjective cognitive decline and mild cognitive impairment. J Alzheimers Dis. 2017:58(3):939-50.

16. van Harten AC, Visser PJ, Pijnenburg YA, et al. Cerebrospinal fluid Abeta42 is the best predictor of clinical progression in patients with subjective complaints. Alzheimers Dement. 2013;9(5):481-7.

17. Tijms BM, Bertens D, Slot RE, et al. Low normal CSF Abeta42 levels predict clinical progression in non-demented subjects. Ann Neurol. 2017:81:749-53.

18. Grill JD, Apostolova LG, Bullain S, et al. Communicating mild cognitive impairment diagnoses with and without amyloid imaging. Alzheimers Res Ther. 2017:9(1):35.

19. Frisoni $G B$, Boccardi $M$, Barkhof F, et al. Strategic roadmap for an early diagnosis of Alzheimer's disease based on biomarkers. Lancet Neurol. 2017; 16(8):661-76.

20. van der Flier WM, Pijnenburg YA, Prins N, et al. Optimizing patient care and research: the Amsterdam Dementia Cohort. J Alzheimers Dis. 2014;41(1):313-27.

21. van der Flier WM, Scheltens P. Amsterdam Dementia Cohort: performing research to optimize care. J Alzheimers Dis. 2018;62(3):1091-111.

22. Jessen F. Subjective and objective cognitive decline at the pre-dementia stage of Alzheimer's disease. Eur Arch Psychiatry Clin Neurosci. 2014;264(Suppl 1):S3-7.

23. Petersen RC, Smith GE, Waring SC, et al. Mild cognitive impairment: clinical characterization and outcome. Arch Neurol. 1999;56(3):303-8.

24. Albert MS, DeKosky ST, Dickson D, et al. The diagnosis of mild cognitive impairment due to Alzheimer's disease: recommendations from the National Institute on Aging-Alzheimer's Association workgroups on diagnostic guidelines for Alzheimer's disease. Alzheimers Dement. 2011;7(3):270-9.

25. McKhann GM, Knopman DS, Chertkow H, et al. The diagnosis of dementia due to Alzheimer's disease: recommendations from the National Institute on Aging-Alzheimer's Association workgroups on diagnostic guidelines for Alzheimer's disease. Alzheimers Dement. 2011;7(3):263-9.

26. Jack CR Jr, Knopman DS, Weigand SD, et al. An operational approach to National Institute on Aging-Alzheimer's Association criteria for preclinical Alzheimer disease. Ann Neurol. 2012;71(6):765-75.
27. Rascovsky K, Hodges JR, Knopman D, et al. Sensitivity of revised diagnostic criteria for the behavioural variant of frontotemporal dementia. Brain. 2011; 134(Pt 9:2456-77.

28. Roman GC, Tatemichi TK, Erkinjuntti T, et al. Vascular dementia: diagnostic criteria for research studies. Report of the NINDS-AIREN International Workshop. Neurology. 1993:43(2):250-60.

29. Patenaude B, Smith SM, Kennedy DN, Jenkinson M. A Bayesian model of shape and appearance for subcortical brain segmentation. Neuroimage. 2011;56(3):907-22

30. Popescu V, Battaglini M, Hoogstrate WS, et al. Optimizing parameter choice for FSL-Brain Extraction Tool (BET) on 3D T1 images in multiple sclerosis. Neuroimage. 2012;61(4):1484-94

31. Scheltens P, Launer LJ, Barkhof F, Weinstein HC, van Gool WA. Visual assessment of medial temporal lobe atrophy on magnetic resonance imaging: interobserver reliability. J Neurol. 1995;242(9):557-60.

32. Pasquier F, Leys D, Weerts JG, et al. Inter- and intraobserver reproducibility of cerebral atrophy assessment on MRI scans with hemispheric infarcts. Eur Neurol. 1996;36(5):268-72.

33. Teunissen CE, Petzold A, Bennett JL, et al. A consensus protocol for the standardization of cerebrospinal fluid collection and biobanking. Neurology. 2009;73(22):1914-22.

34. Kester MI, Boelaarts L, Bouwman FH, et al. Diagnostic impact of CSF biomarkers in a local hospital memory clinic. Dement Geriatr Cogn Disord. 2010;29(6):491-7.

35. Cefalu M. Pointwise confidence intervals for the covariate-adjusted survivor function in the Cox model. Stata J. 2011;11(1):64-81.

36. Crary JF, Trojanowski JQ, Schneider JA, et al. Primary age-related tauopathy (PART): a common pathology associated with human aging. Acta Neuropathol. 2014;128(6):755-66.

37. Ferretti MT, Iulita MF, Cavedo E, et al. Sex differences in Alzheimer disease the gateway to precision medicine. Nat Rev Neurol. 2018;14(8):457-69.

38. Dickerson BC, Stoub TR, Shah RC, et al. Alzheimer-signature MRI biomarker predicts AD dementia in cognitively normal adults. Neurology. 2011;76(16): 1395-402.

39. Jack CR Jr, Knopman DS, Jagust WJ, et al. Tracking pathophysiological processes in Alzheimer's disease: an updated hypothetical model of dynamic biomarkers. Lancet Neurol. 2013;12(2):207-16.

40. Visser PJ, Verhey F, Knol DL, et al. Prevalence and prognostic value of CSF markers of Alzheimer's disease pathology in patients with subjective cognitive impairment or mild cognitive impairment in the DESCRIPA study: a prospective cohort study. Lancet Neurol. 2009;8(7):619-27.

41. Jessen F, Amariglio RE, van Boxtel M, et al. A conceptual framework for research on subjective cognitive decline in preclinical Alzheimer's disease. Alzheimers Dement. 2014;10(6):844-52.

42. Molinuevo JL, Rabin LA, Amariglio R, et al. Implementation of subjective cognitive decline criteria in research studies. Alzheimers Dement. 2017;13(3): 296-311.

43. Slot RER, Sikkes SAM, Berkhof J, et al. Subjective cognitive decline and rates of incident Alzheimer's disease and non-Alzheimer's disease dementia. Alzheimers Dement. 2019;15(3):465-76. https://doi.org/10.1016/j.jalz.2018.10.003.

44. Wang J, Logovinsky V, Hendrix SB, et al. ADCOMS: a composite clinical outcome for prodromal Alzheimer's disease trials. J Neurol Neurosurg Psychiatry. 2016;87(9):993-9.

45. Sikkes SA, Visser PJ, Knol DL, et al. Do instrumental activities of daily living predict dementia at 1- and 2-year follow-up? Findings from the development of screening guidelines and diagnostic criteria for predementia Alzheimer's disease study. J Am Geriatr Soc. 2011;59(12):2273-81.

46. Bratic B, Kurbalija V, Ivanovic M, Oder I, Bosnic Z. Machine learning for predicting cognitive diseases: methods, data sources and risk factors. J Med Syst. 2018;42(12):243

47. Kunneman M, Pel-Littel R, Bouwman FH, et al. Patients' and caregivers' views on conversations and shared decision making in diagnostic testing for Alzheimer's disease: the ABIDE project. Alzheimers Dement. 2017:3:314-22.

48. Roberts JS, Tersegno SM. Estimating and disclosing the risk of developing Alzheimer's disease: challenges, controversies and future directions. Future Neurol. 2010;5(4):501-17.

49. Sevigny J, Chiao P, Bussiere $T$, et al. The antibody aducanumab reduces Abeta plaques in Alzheimer's disease. Nature. 2016;537(7618):50-6.

50. Roberts JS, Dunn LB, Rabinovici GD. Amyloid imaging, risk disclosure and Alzheimer's disease: ethical and practical issues. Neurodegener Dis Manag. 2013;3(3):219-29. 